\title{
Study on Isolation and Radiation Behaviours of a $34 \times 34$ Array-Antennas Based on SIW and Metasurface Properties for Applications in Terahertz Band Over $125-300 \mathrm{GHz}$
}

\author{
Mohammad Alibakhshikenari ${ }^{1 *}$, Bal S. Virdee ${ }^{2}$ \\ ${ }^{1}$ Electronic Engineering Department, University of Rome "Tor Vergata", Via del Politecnico 1, 00133, Rome, ITALY \\ ${ }^{2}$ London Metropolitan University, Center for Communications Technology, School of Computing \& Digital Media, London N7 8DB, UK \\ *alibakhshikenari@ing.uniroma2.it
}

\begin{abstract}
This paper describes the feasibility of conceptual design of a $34 \times 34$ array antenna for operation at Terahertz over a frequency range of $125-300 \mathrm{GHz}$ for $S_{11} \leq-10 \mathrm{~dB}$, which corresponds a fractional bandwidth of $82.35 \%$. Each radiation element constituting the array consists of a square patch of dimensions of $2 \times 2 \mathbf{m m}^{2}$ that are excited by a matched microstrip line. Each patch is isolated from each other with metallic via-holes, which are implemented based on the substrate integrated waveguide (SIW) technique. This technique is shown to effectively reduce mutual coupling between adjacent radiation elements that can otherwise undermine the arrays radiation gain and pattern. Periphery of each patch is embedded with circular dielectric slots, which are created based on the metasurface concept to enhance the radiation gain and efficiency performances. With the proposed approaches (1) the mutual coupling is suppressed on average by $25 \mathrm{~dB}$ over its operating frequency range, and (2) the effective aperture area of the array antenna is extended without increase in the array's physical dimensions. Radiation gain and efficiency of the proposed array antenna over its operating range vary from $7.51 \mathrm{dBi}$ to $40.08 \mathrm{dBi}$, and from $70.51 \%$ to $90.11 \%$, respectively. Improvement in gain and efficiency of approximately $60 \%$ and $30 \%$, respectively, is achieved with the inclusion of the metasurafce slots. The proposed $34 \times 34$ array antenna is proper candidate for applications in Terahertz wireless communication systems.
\end{abstract}

Keywords: Array antennas, Terahertz band, mutual coupling suppression, metasurafce, via-holes, microstrip patch, substrate integrated waveguide (SIW).

\section{INTRODUCTION}

High-volume data communications, such as 8K HD video and high-resolution audio sources, is anticipated in the very near future. This will require near-instantaneous downloads, which makes a speed increase in wireless communication devices vital. This is only possible at Terahertz band. Terahertz communication systems have been extensively investigated, among various Terahertz applications taking full advantage of ultra-wide bandwidths, which enables high speed communications of above $10 \mathrm{Gbit} / \mathrm{s}$ with simple and low spectral efficiency modulation schemes, for example, amplitude shift keying (ASK) or on-off keying (OOK). Studies of prototypes operating at frequencies up to $350 \mathrm{GHz}$ have been reported [1]-[5]. Each of these experimental studies have demonstrated the feasibility of Terahertz communication systems for commercialization. Application of such systems may include fixed wireless access, Terahertz nanocells, WLAN, wireless personal area network, shortrange connecting devices, and board-to-board communications [6].

The disadvantage of Terahertz is the signals at these frequencies attenuate sharply when propagating through space, a highly sensitive receiver is necessary to receive data from weak waves. In addition, to boost the gain of Terahertz system will require high-gain antennas. Although, high-gain antennas can be designed with array antennas they are however inflicted with mutual coupling effects between adjacent radiating elements constituting the array that undermines the array's gain performance. The major challenge is therefore to reduce the mutual coupling in Terahertz array antenna.

It has been shown that mutual coupling or isolation among neighbouring radiating elements in the antenna can be improved by increasing the space between the elements, but this is at the cost of increased antenna size [7]. Although the size of the antenna can be reduced by fabricating it on high dielectric substrate, but the resulting surface waves can significantly deteriorate the radiation characteristics of the antenna. This is because on a finite ground-plane the surface waves are reflected and diffracted at the edges of the substrate, which results in a significant amount of energy loss [8]. Many techniques have been investigated to improve the radiation performance of patch antennas implemented on substrates [9], which include using photonic bandgap (PBG) or electromagnetic bandgap (EBG) structures around the radiating elements [10]. Another approach to suppress surface waves is to use artificial soft and hard surfaces realized with EBG [11]. The soft surface behaves as a perfect electric conductor (PEC) in H-plane and as a perfect magnetic conductor (PMC) in E-plane, and visa-versa for the hard surface. Soft surfaces exhibit bandgaps in only one direction and are created on the ground-plane of a microstrip patch antenna. These results show that the presence of a via close to a radiating element can affect its resonant frequency. In addition, a large surface area is required to implement EBG structures.

This paper describes two techniques based on substrate integrated waveguide (SIW) and metasurface properties to suppress mutual coupling and increase the radiation performances in a conceptual $34 \times 34$ array antenna designed for Terahertz operation over a frequency range of 125-300 GHz. This is achieved by isolating the antennas with metallic via-holes and embedding circular dielectric slots in the periphery of the patch antennas, respectively. 
The proposed technique is shown to increase the radiation performance in terms of gain and efficiency over its over working frequency band. Furthermore, the crosspolarization achieved is low demonstrating high isolation between radiating elements in the array. The antenna design was analysed and optimized by full-wave electromagnetic solvers based on Method of Moments (MoM) and Finite Element Method (FEM). Mutual coupling suppression improvement is shown to be on average of $25 \mathrm{~dB}$. Improvement in radiation gain and efficiency are $60 \%$ and $30 \%$, respectively. The proposed techniques have no effect on the physical dimensions of the array-antennas.

\section{II. $34 \times 34$ ARRAY ANTENNA DESIGN WITH HIGH ISOLATION AND HIGH RADIATION PERFORMANCES}

Common materials used for printed-circuit substrates for high-frequency waves are ceramic, quartz, or Teflon, but when used in the Terahertz band, there is significant signal attenuation and loss of receiving sensitivity. To minimise loss at Terahertz polyimide substrate was employed here. Structure of the $1 \times 2$ referenced array-antennas design is shown in Fig. 1(a), where the square metallic patch structures are patterned on the top side of the dielectric substrate. The antenna's elements are excited through standard aperture coupling illustrated in Fig. 1(b). To realize aperture coupling a rectangular aperture was cut out in the ground-plane under each patch, and a microstrip feed-line etched on the bottom of aperture is used to excite the antenna. The amount of coupling from the feed-line to the patch was optimised by controlling the size of the aperture. Each patch is excited through an aperture under it using a common matched microstrip line. The microstrip line under each column of patch in the array, shown in Fig. 1 (b), branches out from a single waveguide port. With this approach the patches are fed in series. We chose the Copper with electric conductivity $\sigma=5.8 \times 10^{7} \mathrm{~S} / \mathrm{m}$ as metallic pattern and the polyimide with a relative permittivity of $\varepsilon_{r}=3.5$ as dielectric layer. Dimension of the patch is $2 \times 2 \mathrm{~mm}^{2}$ and microstrip line is $0.5 \times 0.2 \mathrm{~mm}^{2}$. The referenced array antenna was used in the construction of the $34 \times 34$ array configuration.

To reduce mutual coupling between adjacent radiating elements it was necessary block surface wave propagation. This was achieved by isolating individual radiating elements with metallic via-holes that were connected directly to the underside ground-plane, as shown in Fig. 2. To realize these metallic via-holes we have utilized the substrate integrated waveguide properties [12]. The radiation gain and efficiency were enhanced by inserting dielectric slots in the periphery of each patch. These slots have realized based on the metasurface technology that by playing the role of the series left-handed capacitances $\left(C_{L}\right)$ actualize the 2-D metamaterial concepts $[13,14]$. Dimensions of the via-holes and dielectric slots as well as their separation from each other was determined by numerous simulations using two different 3D full-wave electromagnetic simulation tools (CST Microwave Studio ${ }^{\circledR}$ and HFSSTM) on a CPU/GPU with 18 GB RAM. In the simulation process, the propagation wave vector $(k)$ was perpendicular to the structure plane whereas the electric field $(\mathrm{E})$ and magnetic field $(\mathrm{H})$ were parallel to the incident plane. The periodic boundary conditions were set along the $x-y$ plane, while the open boundary conditions were chosen along the $z$ plane.

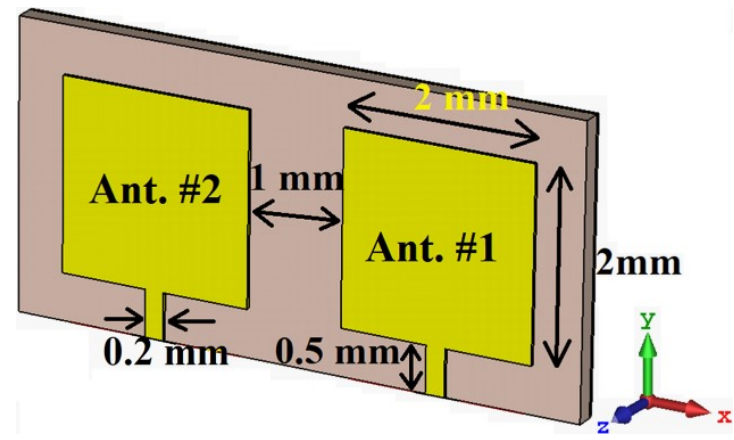

(a)

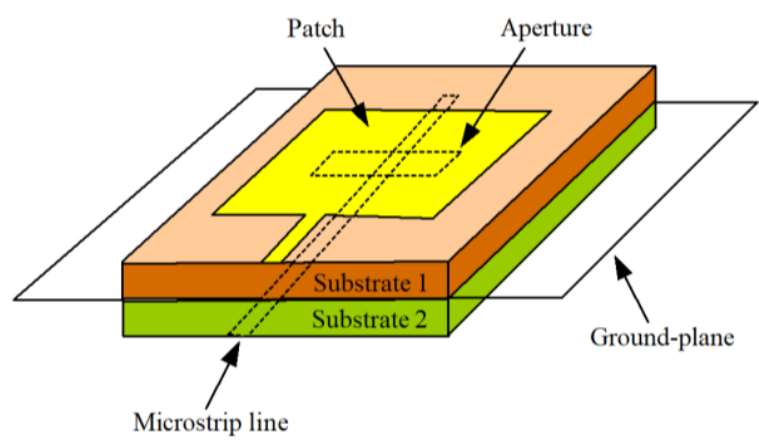

(b)

Fig.1. (a) Reference $1 \times 2$ array antenna with structural parameters, and (b) feed mechanism based on the standard aperture coupling.

Configuration of the proposed $34 \times 34$ array antenna based on the SIW and metasurface technologies is shown in Fig.2. For more clarity, Fig. 2(a) shows a zoomed view of the $34 \times 34$ array located in center of the structure. The optimized separation between each via-holes is $0.1 \mathrm{~mm}$. In the simulation the antennas are fed through a waveguide port. The overall structure of the $34 \times 34$ array antennas is shown in Fig. 2(b). As the array is a symmetrical structure the symmetry option in the solvers was used to decrease computational time.

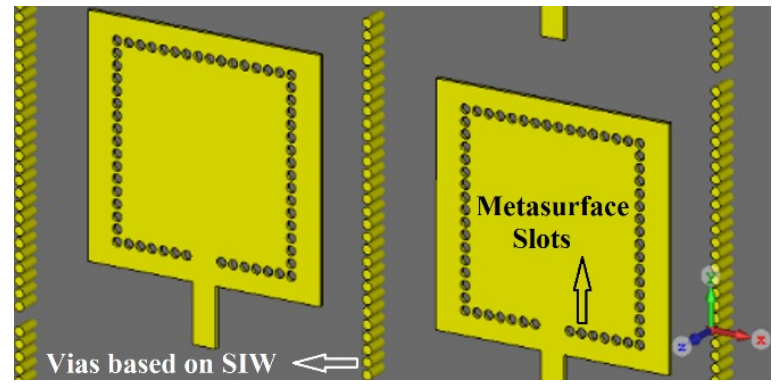

(a) Zoomed view of $34 \times 34$ array antennas to show $1 \times 2$ array elements located in the center 


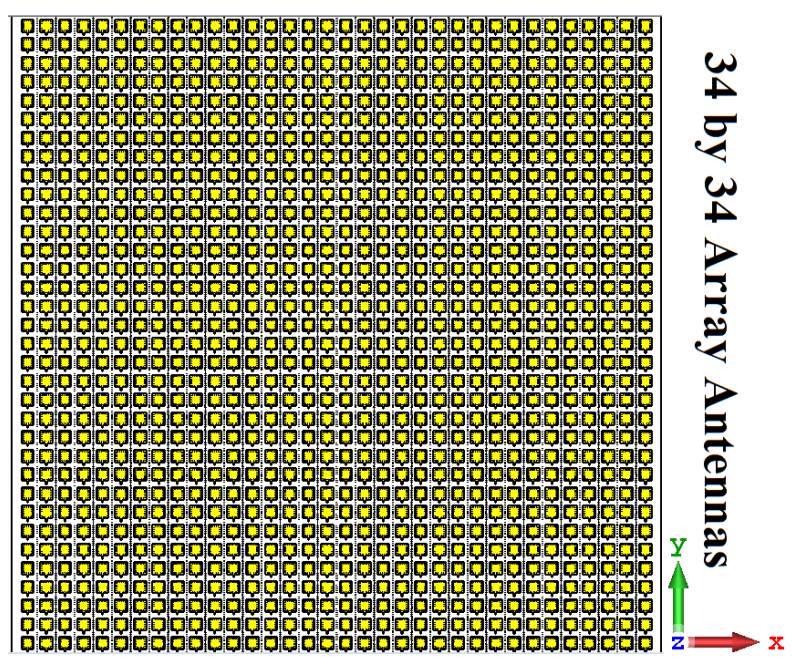

(b) Layout view of whole $34 \times 34$ array antennas

Fig.2. Configuration of the proposed $34 \times 34$ array antennas.

Fig.3 shows the S-parameter responses of the referenced and proposed $34 \times 34$ array antennas without and with metallic the via-holes. This graph shows the proposed array operates over a very wide Terahertz band from 125 $\mathrm{GHz}$ to $300 \mathrm{GHz}$ for $\mathrm{S}_{11} \leq-10 \mathrm{~dB}$, which corresponds to $82.35 \%$ bandwidth. It also clearly shows dramatic improvement in isolation with the via-holes. After implementing the via-holes the minimum, average, and maximum improvement in mutual coupling suppression between the array elements is $5 \mathrm{~dB}, 25 \mathrm{~dB}$, and $50 \mathrm{~dB}$, respectively. There is excellent correlation between CST Microwave Studio ${ }^{\circledR}$ and HFSSTM results. CST Microwave Studio ${ }^{\circledR}$ uses Method of Moments (MoM) to arrive at the solution whereas HFSS ${ }^{\text {TM }}$ uses Finite Element Method (FEM).

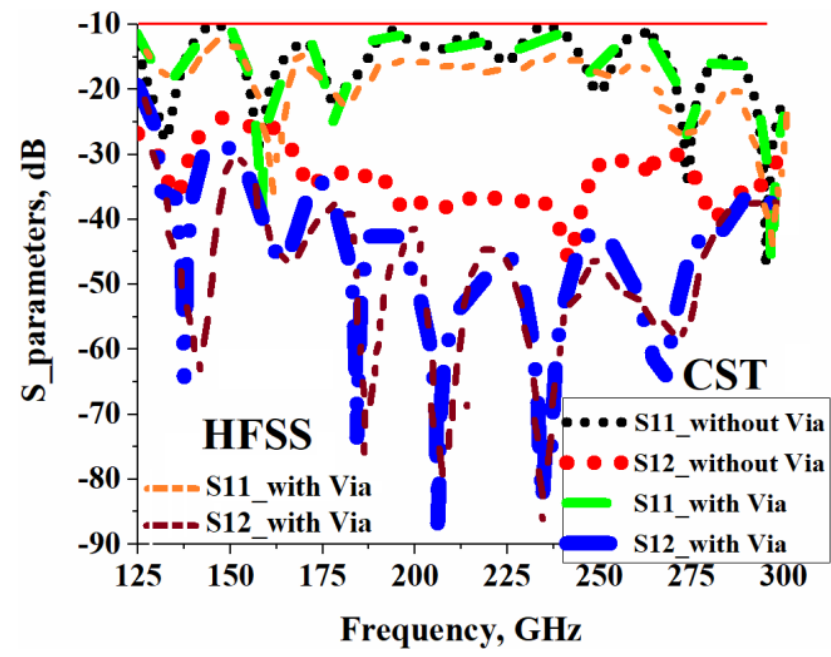

Fig.4. S-parameter responses of the referenced and proposed $34 \times 34$ array antennas with no and with metallic via-holes using two different commercially available $3 \mathrm{D}$ full wave electromagnetics simulation tools (CST Microwave Studio ${ }^{\circledR}$ and HFSS ${ }^{\mathrm{TM}}$ ).

$3 \mathrm{D}$ radiation-gain plots of the proposed $1 \times 2$ array antennas shown in Fig. 2(a) at various operating frequencies after applying the metasurface slots is exhibited in Fig.4. It shows that at lower frequency range between $125 \mathrm{GHz}$ and $200 \mathrm{GHz}$ the beamwidth of the array is much larger than at higher frequencies between $250 \mathrm{GHz}$ and $300 \mathrm{GHz}$ where array is more unidirectional.
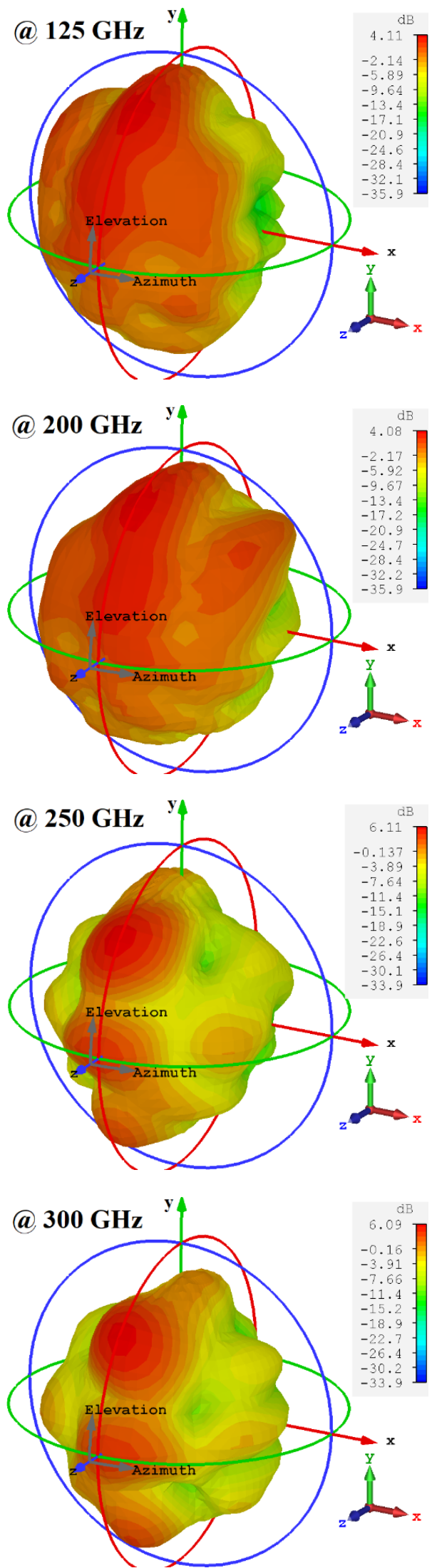

Fig.4. 3-D radiation polar gain plots of the $1 \times 2$ array antenna shown in Fig. 2(a) at various operational frequencies of 125, 200, 250, and 300 $\mathrm{GHz}$. 
Radiation characteristics of the array antennas were improved by inserting circular dielectric slots in the periphery of each patch, as shown in Fig. 2(a). Slots in the patch structure essentially create series left-handed capacitances $\left(C_{L}\right)$ as one of the main structural components of the 2-D metamaterial transmission-lines so-called as "metasurface". By optimizing the number of slots, their diameter, and separation from each other is shown below to effectively increase the array's aperture and hence gain and efficiency performance. This is achieved without enlarging the size of the antenna.

Radiation gain and efficiency responses of the referenced and proposed $34 \times 34$ array antennas with no and with dielectric slots at an angle normal to the plane of the array are shown in Fig.5 in the direction of the main beam. Without the metasurface slots the gain varies from $3.96 \mathrm{dBi}$ to $30.71 \mathrm{dBi}$, and the efficiency from $50.96 \%$ to $75.71 \%$. With application of the metasurface slots the gain now varies from $7.51 \mathrm{dBi}$ to $40.08 \mathrm{dBi}$ and the efficiency from $70.51 \%$ to $90.11 \%$. These results are summarized in Table I, which reveal with metasurface slots minimum gain and efficiency improvements are $90 \%$ and $40 \%$, respectively; and maximum improvements are $\sim 35 \%$ and $20 \%$, respectively.
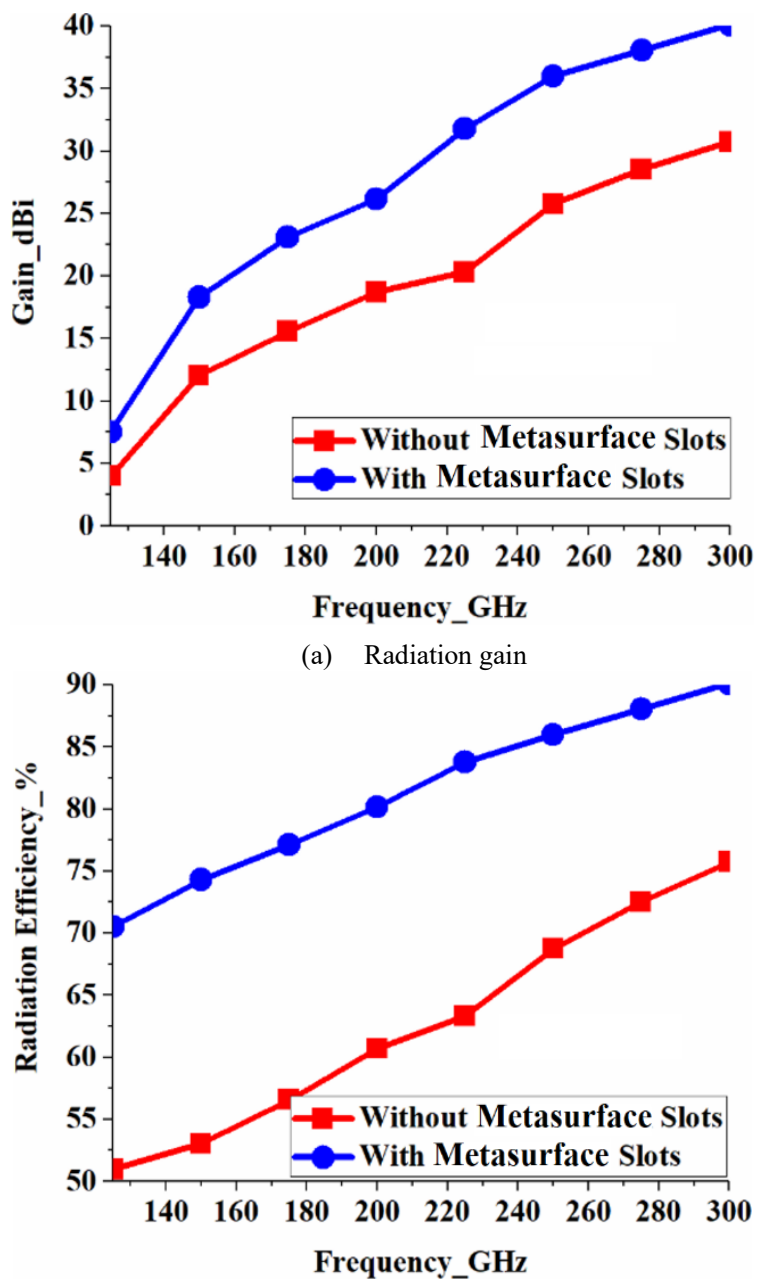

(b) Radiation efficiency

Fig.5. Radiation gain and efficiency of the referenced and proposed $34 \times 34$ array antennas before and after applying the dielectric metasurface slots at the broadside angle.
TABLE I. Radiation Gain and Efficiency With and Without Slots

\begin{tabular}{|c|c|}
\hline \multicolumn{2}{|c|}{ Gain } \\
\hline Minimum without MTM slots & $3.96 \mathrm{dBi}$ \\
\hline Minimum with MTM slots & $7.51 \mathrm{dBi}$ \\
\hline Improvement & $\mathbf{3 . 5 5} \mathbf{~ d B i}$ \\
\hline
\end{tabular}

\begin{tabular}{|c|c|}
\hline \multicolumn{2}{|c|}{ Gain } \\
\hline Maximum without MTM slots & $30.71 \mathrm{dBi}$ \\
\hline Maximum with MTM slots & $40.08 \mathrm{dBi}$ \\
\hline Improvement & $\mathbf{9 . 3 7} \mathbf{~ d B i}$ \\
\hline
\end{tabular}

\begin{tabular}{|c|c|}
\hline \multicolumn{2}{|c|}{ Efficiency } \\
\hline Minimum without MTM slots & $50.96 \%$ \\
\hline Minimum with MTM slots & $70.51 \%$ \\
\hline Improvement & $\mathbf{1 9 . 5 5 \%}$ \\
\hline
\end{tabular}

\begin{tabular}{|c|c|}
\hline \multicolumn{2}{|c|}{ Efficiency } \\
\hline Maximum without MTM slots & $75.71 \%$ \\
\hline Maximum with MTM slots & $90.11 \%$ \\
\hline Improvement & $\mathbf{1 4 . 4 0 \%}$ \\
\hline
\end{tabular}

The co- and cross-polarized radiation gain patterns of the referenced and proposed $34 \times 34$ array antennas without (WO) and with (W) dielectric metasurafce slots in E- and $\mathrm{H}$-planes as a function of broadside angle $(z-x)$ with reference to normal to the plane of the array at the operational frequencies are plotted in Fig.6. It is evident that after applying the metasurface slots the crosspolarization levels significantly drop and co-polarization patterns improve. The gain variation is attributed to phase variation between the elements in the array.
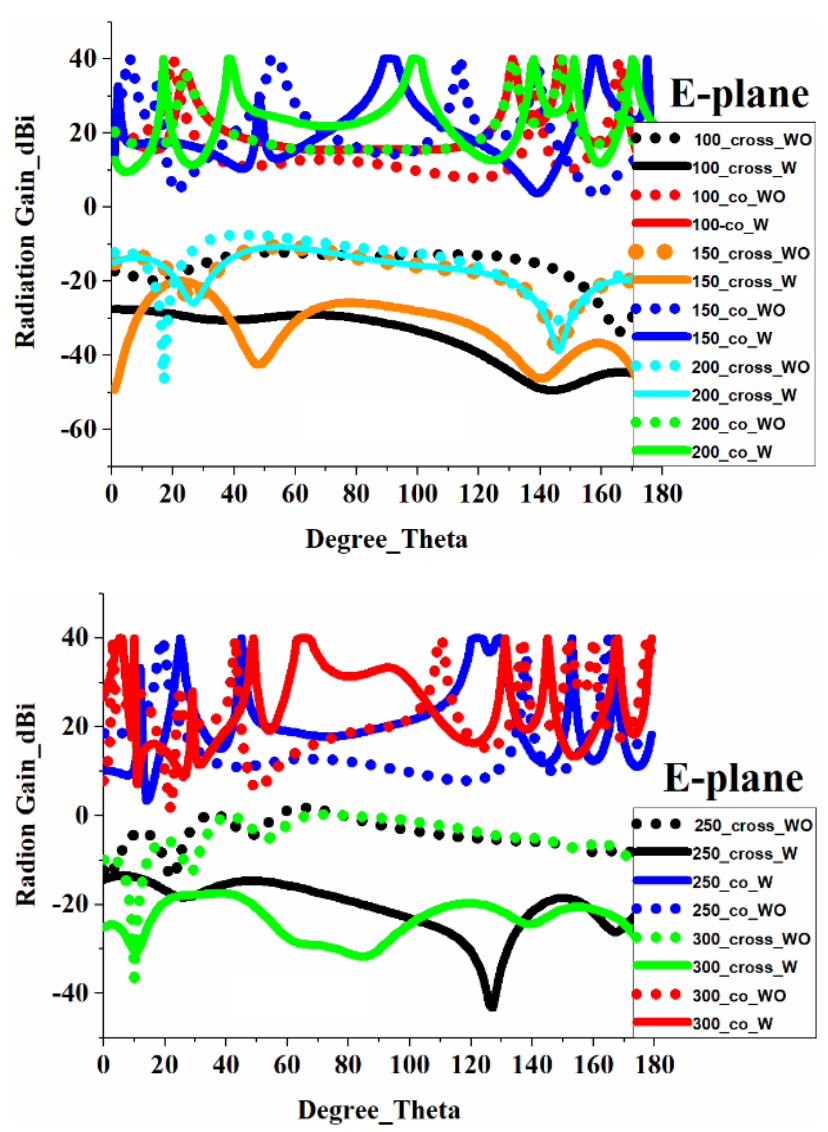

(a) E-plane 

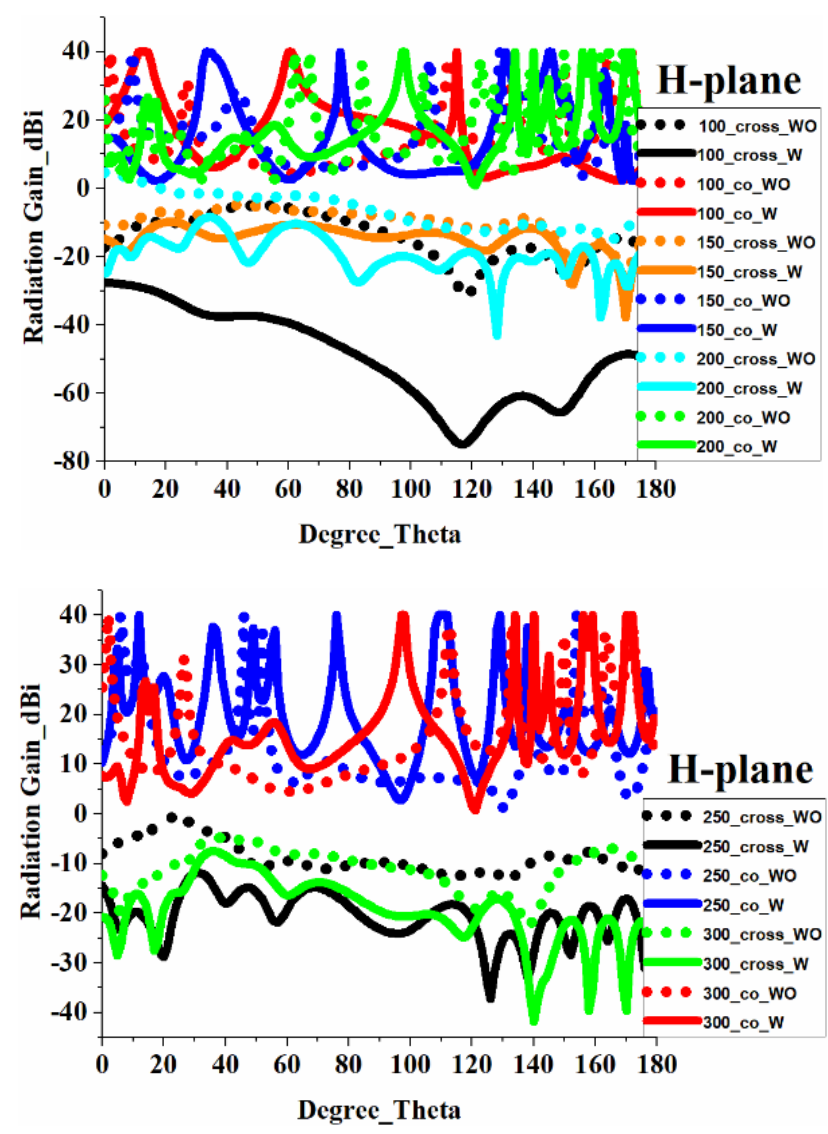

(b) H-plane

Fig.6. Co- and Cross-polarized radiation gain patterns of the referenced and proposed $34 \times 34$ array antennas without $(\mathrm{WO})$ and with $(\mathrm{W})$ metasurface slots in E- and H-planes as a function of broadside angle $(z-$ $x$ ) normal to the plane of the array at various operating frequencies.

Surface current density distributions of the referenced and proposed $1 \times 2$ array antennas shown in Figs. 1(a) and 2(a) without and with metal via-holes are shown in Fig. 7. This figure shows that the via-holes are an effective EM band-gap structure to significantly block surface currents interacting with adjacent radiation elements and thereby effecting the far-field of the antenna array.

\section{COMPARISON OF THE PROPOSED DECOUPLING METHOD WITH THE RECENT MUTUAL COUPLING SUPPRESSION THECNIQUES}

The proposed isolation method is compared with the recent publications employing various mutual coupling suppression techniques. Most of the arrays listed in Table II employ defected ground structures (DGS) to complement their suppression technique to increase isolation between the radiation elements. The radiation pattern of prior techniques is distorted. Here, the array antenna with the proposed isolation method based on SIW and dielectric metasurface slots in each patch has advantage the of: (i) symmetry; (ii) simple design; (iii) improved radiation patterns; (iv) enhanced radiation gain and efficiency; (vi) low cross-polarization levels; and (vii) mutual coupling suppression on average of $25 \mathrm{~dB}$ over its operating band.

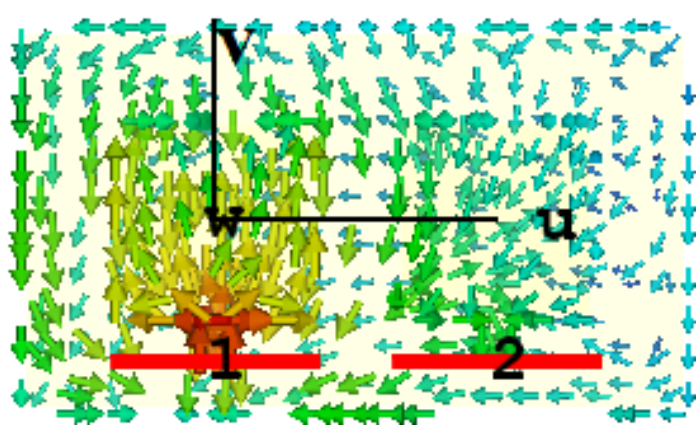

Port\#1 is excited and port\#2 terminated in $50 \Omega$ load.

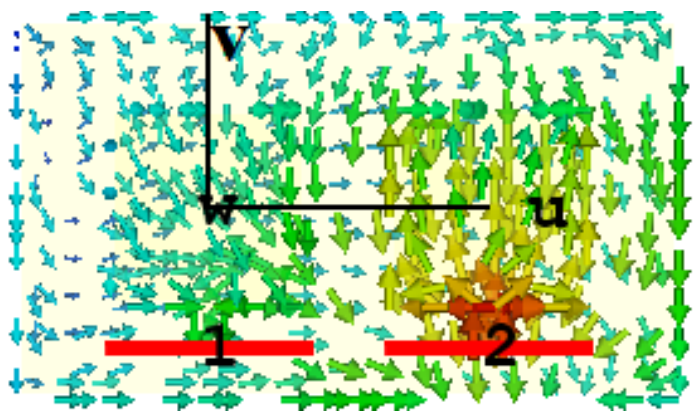

Port\#2 is excited and port\#1 terminated in $50 \Omega$ load.

(a) Without metallic via-holes

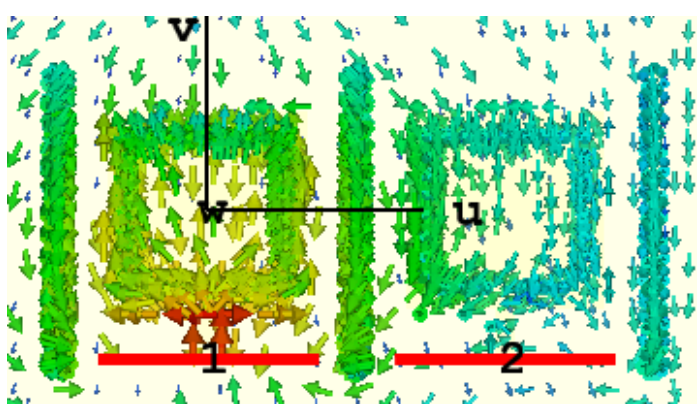

Port\#1 is excited and port\#2 terminated in $50 \Omega$ load.

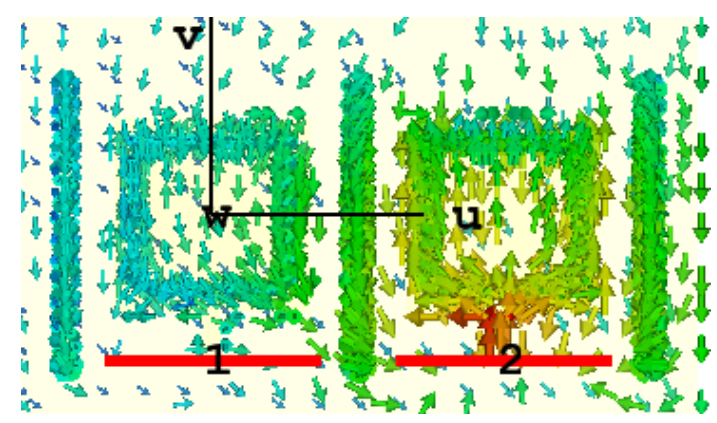

Port\#2 is excited and port\#1 terminated in $50 \Omega$ load.

(b) With metallic via-holes

Fig.7. Surface current density distributions of the referenced and proposed array antennas without and with metal via-holes at $250 \mathrm{GHz}$ for $1 \times 2$ array antennas located in middle of $34 \times 34$ array structure. 
TABLE II. Comparison between the proposed decoupling method with other techniques in literature

\begin{tabular}{|c|c|c|c|c|c|c|}
\hline Ref. & Method & $\begin{array}{c}\text { Max. isolation } \\
\text { improvement }\end{array}$ & $\begin{array}{c}\text { Rad. gain } \\
\text { pattern } \\
\text { deterioration }\end{array}$ & $\begin{array}{c}\text { Application } \\
\text { of DGS }\end{array}$ & $\begin{array}{c}\text { Edge-to- } \\
\text { Edge } \\
\text { Gap }\end{array}$ & $\begin{array}{c}\text { Symmetry } \\
\text { and } \\
\text { Simplicity }\end{array}$ \\
\hline$[15]$ & SCSRR & $10 \mathrm{~dB}$ & Yes & Yes & $0.25 \lambda_{0}$ & No \\
\hline$[16]$ & SCSSRR & $14.6 \mathrm{~dB}$ & Yes & Yes & $0.125 \lambda_{0}$ & No \\
\hline$[17]$ & Compact EBG & $17 \mathrm{~dB}$ & Yes & Yes & $0.8 \lambda_{0}$ & No \\
\hline$[18]$ & Fractal MTM-EMBG & $37 \mathrm{~dB}$ & No & No & $0.5 \lambda_{0}$ & Yes \\
\hline$[19]$ & Meander Line & $10 \mathrm{~dB}$ & No & Yes & $0.055 \lambda_{0}$ & No \\
\hline$[20]$ & EBG & $8.8 \mathrm{~dB}$ & - & Yes & $0.75 \lambda_{0}$ & No \\
\hline$[21]$ & EBG & $13 \mathrm{~dB}$ & Yes & Yes & $0.5 \lambda_{0}$ & No \\
\hline$[22]$ & MTM-DS & $57 \mathrm{~dB}$ & No & No & $0.66 \lambda_{0}$ & Yes \\
\hline$[23]$ & Fractal load DGS & $16 \mathrm{~dB}$ & No & Yes & $0.22 \lambda_{0}$ & No \\
\hline$[24]$ & Slotted Meander-Line & $16 \mathrm{~dB}$ & Yes & Yes & $0.11 \lambda_{0}$ & No \\
\hline$[25]$ & Slots & $>26 \mathrm{~dB}$ & No & No & $0.37 \lambda_{0}$ & Yes \\
\hline$[26]$ & I-Shaped Resonator & $30 \mathrm{~dB}$ & Yes & Yes & $0.45 \lambda_{0}$ & No \\
\hline$[27]$ & W/g MTM & $18 \mathrm{~dB}$ & No & Yes & $0.093 \lambda_{0}$ & No \\
\hline$[28]$ & MSWI & $13.5 \mathrm{~dB}$ & No & No & $1.167 \lambda_{0}$ & Yes \\
\hline $\begin{array}{c}\text { This } \\
\text { work }\end{array}$ & SIW \& Metasurface & $\mathbf{5 0 ~ d B}$ & No & No & $\mathbf{0 . 4 1} \lambda_{0}$ & Yes \\
\hline
\end{tabular}

\section{CONCLUSION}

Simple and effective technique based on the substrate integrated waveguide (SIW) has been presented to reduce unwanted mutual coupling between the radiating elements in $34 \times 34$ array antennas operating at Terahertz band. This is realized by inserting metallic via-holes between the radiating elements to block propagating surface waves. Also, described is a technique utilizing the metasurafce properties to enhance the array's radiation gain and efficiency performances. This was achieved by embedding circular dielectric slots in the periphery of the radiating structure. The proposed techniques do not compromise the antenna size. The proposed array with high isolation and enhanced radiation gain and efficiency performances should facilitate Terahertz communications.

\section{ACKNOWLEDGMENTS}

This work is partially supported by innovation programme under grant agreement H2020-MSCA-ITN-2016 SECRET-722424 and the financial support from the UK EPSRC under grant EP/E022936/1.

\section{REFERENCES}

[1] H. J. Song et al., "Terahertz Wireless Communication Link at 300 GHz," IEEE Topical Meeting Microw. Photonics (MWP), Oct. 2010, pp. 42-45.

[2] M. Abbasi et al., "Single-Chip 220-GHz Active Heterodyne Receiver and Transmitter MMICs With On-Chip Integral Antenna," IEEE Trans. Microw. Theory Techniques, vol. 59, no. 2, Feb. 2011, pp. 466-478.

[3] C. Jastrow et al., "Wireless Digital Data Transmission at $300 \mathrm{GHz}$," Electron. Lett., vol. 46, no. 9, Apr. 2010, pp. 661-663.

[4] H. J. Song et al., "Terahertz Wireless Communication Link at 300 GHz," IEEE Topical Meeting Microw. Photonics (MWP), Oct. 2010, pp. 42-45.

[5] H. J. Song et al., "8 Gbit/s Wireless Data Transmission at $250 \mathrm{GHz}$," Electron. Lett., vol. 45, no. 22, Oct. 2009, pp. 1121-1122.

[6] H. J. Song and T. Nagatsuma, "Present and Future Terahertz Communications," IEEE Trans. THz Sci. Technol., vol. 1, no. 1, Sept. 2011, pp. 256-263.

[7] Y. Gou, S. Yang, Q. Zhu, and Z. Nie, "A Compact Dual-Polarized Double-Shaped Patch Antenna with High Isolation," IEEE Trans. Ant and Propag., Vol. 61, No. 8, 4349-4353, 2013.

[8] S. Li, X. Cao, J. Gao, and P. Gao, "High-Isolation Dual-Polarized Microstrip Antenna Via Substrate Integrated Waveguide Technology," Radioengineering, Vol. 23, No. 4, 1092-1098, 2014.
[9] X.-J. Lin, Z.-M. Xie, and P.-S. Zhang, "High Isolation Dual-Polarized Patch Antenna with Hybrid Ring Feeding," Int. Journal of Antennas and Propagation, Vol. 2017, No. 3, 1-6, 2017.

[10] M. Alibakhshikenari, M. Vittori, S. Colangeli, B. S. Virdee, A. Andujar, J. Anguera, and E. Limiti, "EM Isolation Enhancement Based on Metamaterial Concept in Antenna Array System to Support FullDuplex Application," IEEE Asia Pacific Microwave Conf. Nov. 2017.

[11] P. S. Kildal, A. A. Kishk, and S. Maci, "Special Issue on Artificial Magnetic Conductors, Soft/Hard Surfaces, and Other Complex Surfaces,' IEEE Trans. Ant. And Propag., Vol. 53, No. 1, Part 1, 2-7, 2005.

[12] Ke Wu, Yu Jian Cheng, Tarek Djerafi, and Wei Hong,"SubstrateIntegrated Millimeter-Wave and Terahertz Antenna Technology", Proceedings of the IEEE, vol. 100, no. 7, pp. 2219-2232, July 2012

[13] Mohamed El Badawe, Thamer S. Almoneef \& Omar M. Ramahi, "A True Metasurface Antenna”, Scientific Reports volume 6, 19268 (2016). [14] Niamat Hussain and Ikmo Park,"Design of a wide-gain-bandwidth metasurface antenna at terahertz frequency", AIP Advances 7, 055313 (2017).

[15] M. M. B. Suwailam, O. F. Siddiqui, and O. M. Ramahi, "Mutual coupling reduction between microstrip patch antennas using slottedcomplementary split-ring resonators," IEEE Antennas \& Wireless Propag., Lett., vol. 9, pp. 876-878, 2010.

[16] M. F. Shafique, Z. Qamar, L. Riaz, R. Saleem, and S. A. Khan, "Coupling suppression in densely packed microstrip arrays using metamaterial structure," Microwave and Optical Technology Letters, vol. 57, No. 3, pp. 759-763, 2015.

[17] M. T. Islam, and M. S. Alam, "Compact EBG Structure for Alleviating Mutual Coupling Between Patch Antenna Array Elements," Progress in Electromagnetics Research, vol. 137, pp. 425-38, 2013.

[18]M. Alibakhshikenari, B. S. Virdee, C. H. See, R. A. Abd-Alhameed, A. H. Ali, F. Falcone, E. Limiti, "Study on Isolation Improvement Between Closely Packed Patch Antenna Arrays Based on Fractal Metamaterial Electromagnetic Bandgap Structures," IET Microwaves, Antennas \& Propagation, Volume 12, Issue 14, 28 November 2018, p. $2241-2247$.

[19] J. Ghosh, S. Ghosal, D. Mitra, and S. R. B. Chaudhuri, "Mutual Coupling Reduction Between Closely Placed Microstrip Patch Antenna Using Meander Line Resonator," Prog. in Electromag. Research Lett., vol. 59, pp. 115-122, 2016.

[20] F. Yang and Y. Rahmat-Samii, "Microstrip Antennas Integrated with Elctromagnetic Band-Gap (EBG) Structures: A Low Mutual Coupling Design for Array Applications," IEEE Trans. Antennas Propag, vol. 51, no. 10 , pp. 2936-2946, Oct. 2003.

[21] M. J. Al-Hasan, T. A. Denidni, and A. R. Sebak, "Millimeter Wave Compact EBG Structure for Mutual Coupling Reduction Applications," IEEE Trans. Antennas Propag., vol. 63, no. 2, pp. 823-828, Feb. 2015.

[22] M. Alibakhshikenari, B. S. Virdee, C. H. See, R. A. Abd-Alhameed, M. Khalily, F. Falcone, E. Limiti, "Interaction Between Closely Packed Array Antenna Elements Using Metasurface for Applications Such as MIMO Systems and Synthetic Aperture Radars," Radio Science, Volume 53, Issue 11, November 2018, Pages 1368-1381. 
[23] X. Yang, Y. Liu, Y.-X. Xu, and S.-X. Gong, "Isolation Enhancement in Patch Antenna Array with fractal UC-EBG Structure and Cross Slot", IEEE Antennas \& Wireless Propag. Lett., vol. 16, 2017, pp. 2175-2178.

[24] M. Alsath, G., M. Kanagasabai, and B. Balasubramanian, "Implementation of Slotted Meander Line Resonators for Isolation Enhancement in Microstrip Patch Antenna Arrays," IEEE Ant. \& Wireless Propag. Letters, vol. 12, pp. 15-18, 2013.

[25]M. Alibakhshikenari, B. S. Virdee, P. Shukla, C. H. See, R. AbdAlhameed, M. Khalily, F. Falcone, E. Limiti, “Antenna Mutual Coupling Suppression Over Wideband Using Embedded Periphery Slot for Antenna Arrays," $\quad$ Electronics, $\quad 2018, \quad 7(9), \quad 198$ https://doi.org/10.3390/electronics7090198.

[26] C. K. Ghosh, and S. K. Parui, "Reduction of Mutual Coupling Between E-Shaped Microstrip Antennas by Using a Simple Microstrip ISection," Microwave \& Optical Tech. Lett., vol. 55, no. 11, pp. 2544 $2549,2013$.

[27] Z. Qamar, and H. C. Park, "Compact Waveguided Metamaterials for Suppression of Mutual Coupling in Microstrip Array," Progress in Electromagnetic Research, vol. 149, pp. 183-192, 2014.

[28]M. Alibakhshikenari, B. S. Virdee, P. Shukla, C. H. See, R. AbdAlhameed, F. Falcone, and E. Limiti, "Meta-surface Wall Suppression of Mutual Coupling between Microstrip Patch Antenna Arrays for THz-band Applications," Progress in Electromagnetics Research Letters, Vol. 75, page 105-111, 2018. 